\title{
Segmentation of Melanoma Skin Lesions Using Anisotropic Diffusion and Adaptive Thresholding
}

\author{
Adil H. Khan \\ Ghazanfar Latif \\ Universiti Malaysia Sarawak, Malaysia. \\ Universiti Malaysia Sarawak, Malaysia. \\ Prince Mohammad bin Fahd University, \\ Saudi Arabia. \\ Prince Mohammad bin Fahd University, \\ Saudi Arabia. \\ akhan@pmu.edu.sa \\ glatif@pmu.edu.sa \\ D. N. F. Awang Iskandar \\ Universiti Malaysia Sarawak, Malaysia. \\ dnfaiz@unimas.my \\ Jaafar Alghazo \\ Mohsin Butt \\ Prince Mohammad bin Fahd University, Saudi Arabia. \\ Prince Mohammad bin Fahd University, Saudi Arabia. \\ King Fahd University of Petroleum and Minerals, Saudi Arabia King Fahd University of Petroleum and Minerals, Saudi Arabia

$$
\text { jghazo@pmu.edu.sa }
$$ \\ Arabia.mohsinbutt@kfupm.edu.sa
}

\begin{abstract}
Segmentation is the first and most important task in the diagnosis of skin cancer using computer-aided systems and due to complex structure of skin lesions, the automated process may lead to a completely different diagnosis. In this paper, a novel segmentation method of skin lesions is proposed which is both effective and simple to implement. Smoothing of skin lesions in original image plays a pivotal role to secure an accurate segmented image. Anisotropic Diffusion Filter (ADF) is used in the initial stage to smooth images with preserved edges. Adaptive thresholding is then applied to segment the skin lesion of the image by binarizing it. The morphological operations are applied for further enhancement and final segmented image is obtained by applying proposed boundary conditions in which objects are selected on basis of distance. The proposed technique is tested on over 300 images and averaged results are compared with existing methods like L-SRM, Otsu-R, Otsu-RGB and TDLS. The proposed method achieved an average accuracy of $96.6 \%$. Visual results for selected images also depicted better performance of proposed method even in the presence of bad illumination and rough skin lesions in the image.
\end{abstract}

\section{CCS Concepts}

- Computing methodologies $\rightarrow$ Image segmentation

\section{Keywords}

Melanoma Skin Lesions; Segmentation; Anisotropic Diffusion; Skin Cancer; Adaptive Thresholding.

\section{INTRODUCTION}

Melanoma is a type of skin cancer that originates from small pigments on the skin of subjects. The pigments contain cells that called melanocytes. Fatality rates caused by melanoma skin

Permission to make digital or hard copies of all or part of this work for personal or classroom use is granted without fee provided that copies are not made or distributed for profit or commercial advantage and that copies bear this notice and the full citation on the first page. Copyrights for components of this work owned by others than ACM must be honored. Abstracting with credit is permitted. To copy otherwise, or republish, to post on servers or to redistribute to lists, requires prior specific permission and/or a fee. Request permissions from Permissions@acm.org.

ICBET '18, April 23-25, 2018, Bali, Indonesia

(C) 2018 Association for Computing Machinery.

ACM ISBN 978-1-4503-6369-3/18/04 ...\$15.00

https://doi.org/10.1145/3208955.3208961 lesions are the highest amongst skin cancer patients. Melanoma skins cancer has been on the rise for the past few year [1]. The dermatologists need to screen every patient thoroughly for possible infection. Image processing techniques can help the dermatologists by automatically filtering the melanoma affected patients.

A dermatoscope is a device which is used to capture different skin conditions. The device uses a magnifier which is illuminated by a light input which helps in capturing the color and microstructures of skin lesions more accurately. The images obtained from a dermatoscope are called dermoscopy images. Currently, most of the computer automated systems use the dermoscopy images for analyzing and diagnosing skin cancer patients. However, these methods are still in their preliminary stage to be used by dermatologists in a clinical environment [2]. In recent years, the use of smartphone cameras to promote health care and assist in early detection of the skin lesions. Various applications in smartphones have potential to use these images for review by a dermatologist or automated detection of the skin lesions [3].

Pre-processing is an important step in every image segmentation technique. Various image processing techniques can be used in the pre-processing stage to help remove redundant data from the input image. Image smoothing and denoising is one of the essential pre-processing methods which is used to remove additive noise from the image that is introduced during the image capturing process. Different linear and non-linear filtering methods to de-noise the images have been suggested. In linear filtering approach, e.g. Averaging, Gaussian filtering etc., every image pixel is assigned a value based on a linear weighted relation with the surrounding pixels. Gaussian filtering uses a Gaussian function to transform the image pixels and smooth the image for segmentation. Most of the linear filtering approaches end up in the removal of the image noise but degrade the edge information as well. Anisotropic Diffusion Filtering (ADF) is a non-linear image smoothing and denoising method in which image quality is not degraded after the removal of noise and edge details are also kept accurately for further processing in the segmentation phase [4-5]. The method ensures the fine details in the image are kept while removing the noise using causality and smoothing parameters.

Image Segmentation is a method used in image processing to partition an image into different regions and extract meaningful information. In dermoscopy images, segmentation can be used to identify the boundary of the skin lesions that may lead to cancer. Various image features are extracted from the segmented image that can be used to classify the image as cancerous or non- 